Session 3232

\title{
Emerging Classroom Technology: Opportunities and Pitfalls
}

\author{
David G. Meyer \\ School of Electrical \& Computer Engineering/Purdue University
}

\begin{abstract}
Based on the premise that currently available technology can and should be better utilized to enhance the classroom experience - and ultimately to help students learn, this paper outlines a series of opportunities and pitfalls associated with deploying technology both inside and outside the classroom. Issues addressed include teaching in the visual arena, adapting course material to different learning styles, consolidating lecture presentation materials onto a single medium, using wireless LANs in the classroom, and using technologybased instructional delivery in place of traditional lectures for presentation of selected topics.
\end{abstract}

\section{INTRODUCTION}

The primary motivating objective for deploying technology-based instructional delivery systems is to help students learn. Use of such educational tools allows instructors to: (a) teach in the visual arena; (b) adapt presentation of course material to a variety of different learning styles; (c) make lecture presentation material as well as supplementary course material (both audio \& visual) interactively accessible to students outside of class, in a hierarchical, cross-indexed fashion; (d) modularize course material in a logical fashion; and (e) facilitate non-linear presentation of course material, both in terms of module length and "density" (i.e., number of modules covered in a given period of time). Another motivating objective for utilizing technology-based instructional delivery systems is to make more efficient and effective use of instructional staff. Not only can students' access to instructional resources be enhanced, but also the potential exists for freeing up professors' time for more one-on-one interaction with students.

A common criticism is that we, as engineering professors, seldom use our "own" tools (e.g., high performance graphics workstations and projection display devices) in teaching our students. The on-going challenge, then, is to make better, more effective use of emerging and currently available technology, both inside and outside the classroom. "More" use, however, is not necessarily "better" — inappropriate use of instructional technology can potentially be worse than "no" use of technology (i.e., can actually detract from the learning experience). The focus that must be maintained in utilizing technology is quality of education. The importance of human interaction as an integral part of the educational process must also be kept in perspective.

To help summarize some of the current issues associated with use of instructional technology, this paper outlines a series of opportunities along with possible pitfalls, and concludes with a summary of some of the challenges ahead. 


\section{OUTLINE OF OPPORTUNITIES AND POSSIBLE PITFALLS}

Outlined below are a series of opportunities and possible pitfalls associated with use of instructional technology. They are based on the author's experience gained in deploying instructional multimedia delivery systems $^{1-4}$; in formulating experimental, technology-based course formats ${ }^{5}$; and in serving on a Universitywide Classroom Technology Advisory Committee.

\section{Opportunity \#1:}

Perhaps the greatest opportunity currently available is the ability to effectively teach in the visual arena, i.e., to make increased use of computer-generated text, graphics, and images in classroom presentations. The argument for increased use of visual media in engineering education has been aptly articulated in the literature. $^{6-8}$ Since print dominates our environment and educational methods, students are forced to process information and learn predominantly with the brain's left hemisphere. Most of what engineering students must learn, however, requires them to develop right-brain processing skills. This leads to a fundamental incompatibility in the general learning process, an incompatibility which is particularly acute in engineering education: right-brain processing funneled through left-brain "sequential I/O" (i.e., printed text). In contrast, visual media provide a direct, "parallel I/O" path to the brain's right hemisphere. A good physical analogy is interfacing a computer to a hard disk drive using a serial port versus a direct memory access controller. Clearly, in an increasingly vigorous visual culture, effective use of visual media in engineering education is absolutely essential.

\section{Possible Pitfalls:}

Despite a series of recent advances in video/graphics projection technology, some possible pitfalls remain. First, if the visualizations used in a lecture presentation are not consolidated onto a single medium (storage, access, projection), the "switching overhead" among the various visual sources will be high (e.g., due to the disparity in illumination level associated with an overhead transparency projector vs. a video/graphics projector, and/or changes in room lighting necessary to accommodate the different modes of projection). Second, if the equipment (e.g., video projector and computer) is not built in, the setup/teardown overhead will be high - which will ultimately discourage continued/future use of technology in the classroom. Third, operating equipment under subdued lighting conditions required by today's video projectors — particularly computer keyboards - is difficult, cumbersome, and error-prone. Finally, care must be taken in displaying the "screen" of a high resolution workstation on a video projector in a large room — if the character font is not appropriately sized, the information displayed will be illegible (irregardless of the video/graphics projector's resolution!). Poor visualizations can be produced even with the finest equipment.

\section{Opportunity \#2:}

Another emerging opportunity, that should prove quite useful in the classroom, is the capability to consolidate all lecture presentation materials onto a single "medium" (encompassing storage, access, and projection). This can be accomplished using high performance file/media servers with digital video capability and high bandwidth communication links to computers in the classroom.

\section{Possible Pitfalls:}

Unfortunately, "plug and play" is not yet a reality — a variety of (inherently incompatible) computing platforms and standards (e.g., display formats, compression algorithms) currently exist. Given that: (a) it is 
impractical to support all platforms/standards in a single classroom, and (b) it is impossible to force everyone to use a common platform (e.g., Mac, PC, workstation) — what should be the "equipment of choice" for classroom installation? And, given that choice, how can this "generic" platform (that, by definition, no one likes) be dynamically reconfigured (i.e., between classes) for each instructor's preferred operating environment? One solution would be for each professor to bring his/her own laptop computer to each class (in order to ensure the availability of a familiar computing platform and operating environment), but this is still fairly cumbersome. The bottom line: we're still waiting for a good solution.

\section{Opportunity \#3:}

Another interesting possibility, used experimentally, is use of a wireless LAN in the classroom, to facilitate electronic distribution of lecture presentation material as well as electronic student-professor interaction and student-student collaboration (plus electronic submission of questions, electronic posting of answers, etc.).

\section{Possible Pitfalls:}

A number of hurdles remain before widespread use of wireless classroom LANs will become a reality. The biggest impediment is related to infrastructure: many traditional classrooms would have to be significantly refurbished (e.g., tables instead of desks, power receptacles at each table, etc.) before being suitable for laptop computer use. Another consideration is that students would be required to purchase compatible computing platforms and configure them with similar operating environments. Finally, questions of network extensibility, interference between classrooms, security, protection of intellectual property rights, etc., remain.

\section{Opportunity \#4:}

Another opportunity at our doorstep is the ability to make lecture presentation materials, as well supplementary course materials (both audio and visual), interactively accessible by students outside of class - in a hierarchical, cross-indexed fashion.

\section{Possible Pitfalls:}

Anyone who has attempted to create multimedia tools (or even Web pages) is well aware of the significant learning curve associated with most authoring tools. And since so many different possibilities exist for organizing databases of visual and audio materials (a de facto standard is yet to emerge), it is difficult to decide which authoring tool(s) one should (attempt to) master. Once the authoring tools have been mastered, though, a significant out-of-class development time is required to produce (and maintain) "professionallooking" presentation materials.

Another consideration is video server technology: while it is rapidly developing, it has not yet matured - compression algorithms and specialized network protocols are continuously evolving. Further, digital storage requirements for "an entire course worth of material" (e.g., 40 hours of full-motion, high resolution audio, video, and graphics) are massive — an order of magnitude improvement in digital storage technology is necessary for "all-digital" solutions to be tractable. Tomorrow's "common" digital communication links need to be of significantly higher bandwidth than currently supported by ubiquitous networks such as ethernet. As of yet unanswered questions include how to most effectively back up massive digital audio/visual databases, and how to ensure intellectual property rights (e.g., prevent illegal copying or duplication of a CD-ROM "book"). 


\section{Opportunity \#5:}

Another opportunity instructional technology affords is the ability to adapt presentation of course material to a variety of different learning styles, via interactive instructional delivery systems that allow students to navigate a hierarchical search path through a rich database of instructional (audio/visual) material. For active learners, instructional technology could allow self-motivated pursuit of information. For reflective learners, instructional technology could provide the opportunity to investigate and understand the interrelationships. For sequential learners, instructional technology could provide the opportunity to review the flow of lecture material. And finally for global learners, instructional technology could allow navigation of complete course material under individual direction, yet present a coherent association of previous, current, related, and future material.

\section{Possible Pitfalls:}

The major pitfalls here boil down to time and effort: (a) navigating the learning curve associated with using an audio/visual database management tool; (b) the time and effort required to organize a large database of (audio/visual) instructional material in a hierarchical, interrelated fashion; and (c) the time and effort required to maintain the database of instructional material.

\section{Opportunity \#6:}

An emerging opportunity involves use of desktop videoconferencing and collaboration tools to provide off-campus students (or students with disabilities) with easier, more efficient and effective access to instructional staff (e.g., video office hours, video mail, etc.).

\section{Possible Pitfalls:}

Since most professors are already inundated with (text) E-mail, the prospect of adding video electronic mail may not be that appealing. From a technological viewpoint, many currently available desktop videoconferencing tools require ISDN access (at least for "reasonable" quality motion video), which is not universally available (and relatively expensive compared with conventional telephone lines). From a psychological viewpoint, use of professor-to-student videoconferencing (in place of "office contact") could be viewed as a further "depersonalization" of the educational process.

\section{Opportunity \#7:}

The final issue addressed here is the (selective) use technology-based instructional delivery in place of traditional ("live") lectures, which would offer several potential opportunities: (a) make more effective use of "live lecture" classtime (e.g., eliminate waste of class time reviewing material that only a small portion of the students need); (b) make more efficient and effective utilization of instructional staff (professors and teaching assistants), and thereby free up time for more one-on-one interaction with students; (c) facilitate "just-in-time" delivery of instructional modules (e.g., how to use a particular CAD tool); and (d) facilitate "modularization" of course material that would otherwise be impossible in the traditional "N-times-a-week-for-M-minutes" setting.

\section{Possible Pitfalls:}

This is perhaps the most "controversial" opportunity, for a variety of reasons. The first has to do with culture - some students, for whatever reason, are inextricably ensconced in the "N-times-a-week-for-M- 
minutes-with-a-live-body" class format. The second is based on the major development effort required significant changes in entire curricula would need to be made in order to take full advantage of technologybased instructional delivery. Finally, the "jury is still out" on the effectiveness of technology-based instructional delivery. While there has been much speculation concerning how the "information superhighway" along with advances in computer and communications technology — will impact the "classroom of the future" 9 , few studies have been completed that document how and in what ways learning is affected by use of technology-based delivery systems

\section{SUMMARY AND FUTURE CHALLENGES}

Currently available technology can and should be better utilized to enhance the classroom experience, and ultimately help students learn. Emerging computer and communications technology will further enhance storage, access, and display capabilities in the classroom. Perhaps the most significant challenge is not technological, but rather cultural - that of convincing both students and faculty of the "need to do things differently" in the midst of the information age revolution. Further research is needed to determine how well students learn in "non-traditional" environments, both inside and outside the classroom, and how curricula should change in response to the capabilities afforded by technology-based instructional delivery systems.

\section{REFERENCES}

1. Meyer, D. G. and Zoltowski, C. B., "Use of the Laser Videodisc Medium as an Educational Delivery Tool,' 1989 ASEE Annual Conference Proceedings, pp. 174-176.

2. Meyer, D. G., "'On the Delivery of Visualizations for Teaching Engineering Design Using the Laser Videodisc Medium,' 1991 ASEE Annual Conference Proceedings, pp. 591-594.

3. Meyer, D. G., 'Workshop on Use of the Laser Videodisc Medium as an Educational Delivery Tool,' 1991 FIE Annual Conference Proceedings, West Lafayette, Indiana, September 1991, pp. 14-16.

4. Meyer, D. G., Krzyzkowski, R. A. and Hoefflinger, M., "The VideoJockey System: A Testbed for CostEffective Multimedia Instructional Delivery,', Proceedings of 1992 Frontiers in Education Conference, Nashville, Tennessee, November 1992, pp. 168-172.

5. Meyer, D. G. and Krzyzkowski, R. A., "Experience Using the VideoJockey System for Instructional Multimedia Delivery,' Proceedings of the 1994 Frontiers in Education Conference, San Jose, California, November 1994, pp. 262-266.

6. Felder, R. M. and Silverman, L. K., “Learning and Teaching Styles in Engineering Education,'” Engineering Education, April 1988, pp. 674-681.

7. Waldheim, G. P., "Understanding How Students Understand: A Prerequisite to Planning Effective Teaching-Learning Methods,', Engineering Education, February 1987, pp. 306-308.

8. Murr, L. E., 'In the Visual Culture,' Engineering Education, December 1988, pp. 170-172.

9. House, D., “Cruising Your PC Down the Information Highway,' Innovator, Vol. 7, No. 2, August 1994, pp. 10-12.

\section{BIOGRAPHY}

DAVID G. MEYER is currently an Associate Professor at the School of Electrical \& Computer Engineering, Purdue University, West Lafayette, Indiana, specializing in multimedia educational delivery systems. 